\title{
Homogeneity Hypothesis in the Context of Asset Pricing Models. The Quadratic Market Model.
}

\author{
Giovanni Barone-A desi \\ (Universita' della Svizzera Italiana, Lugano,CH) \\ Patrick Gagliardini \\ (Universita' della Svizzera Italian, Lugano,CH) \\ Giovanni Urga \\ (City University Business School, London, U.K.) ${ }^{\mathrm{y}}$
}

October 29, 2000

\begin{abstract}
A bstract
This paper proposes a two factor model for asset pricing. We formulate a model of asset returns that in addition to the traditional market return term includes also the square of the market return to account for risk originating from coskewness with the market portofolio. The quadratic term is able to account for heterogeneities across portfolios. We conclude that the extra term is signi...cant and that the homogeneity hypothesis is accepted only in the presence of this term.
\end{abstract}

J EL Classi..cation: G12, C23

Keywords: A sset Pricing Models, Panel

\footnotetext{
${ }^{x}$ We wish to thank the participants to the 9th Conference on Panel Data (Geneva, 22-23 $J$ une, 2000) for helpful discussion. M.R ockinger provided very useful comments which helped to improve the paper. We wish to thank R.J agannathan, R.K an, and C.Zhang for providing us with their data set. The usual disclaimer applies.

y C orresponding author: D epartment of Investment, R isk M anagement and Insurance, City University B usines School, Frobisher Crescent, Barbican Centre, London EC2Y 8HB (U.K.). Tel.+/ 44/ 20/74778698. Fax.+/ 44/ 20/74778885. e-mail:g.urga@city.ac.uk
} 


\section{Introduction}

A sset pricing models generally express expected returns on ..nancial assets as linear functions of covariances of returns with some systematic risk factors. Sharpe (1964), Lintner (1965), Black (1972), Merton (1973), K raus and Litzenberger (1976), Ross (1976), Breeden (1979), Barone-A desi (1985), J agannathan and Wang (1996), Harvey and Siddique $(1999,2000)$ have proposed several formulations of this general paradigm. See Campbell (2000) for a recent survey on the ..eld of asset pricing. M ost of the empirical tests to date have produced negative or ambiguous results. These ..ndings have spurred renewed interest in the statistical properties of testing methodologies currently available. Among recent studies, Shanken (1992), K an and Zhang (1999a,b) provide thorough analyses of the statistical methodologies commonly employed and highlight the sources of ambiguity that plague their ..ndings.

It appears that only the preliminary knowledge of the return generating process may lead to the design of reliable tests. Because this condition is never met in practice, researchers are forced to make unpalatable choices between powerful tests that are misleading in the presence of possible model speci..cations or more tolerant tests, such as the ones based on the stochastic discount factor methodology, that lack of power. The ..rst choice may lead not only to the rejection of correct models, but also to the acceptance of irrelevant factors as sources of systematic risk, as noted by K an and Zhang (1999a,b). O n the other hand the choice of the stochastic discount factor methodology fails to discriminate among competing models and leads to very large con..dence intervals for estimated risk premia (Cochrane (1996) and K an and Zhang (1999a,b)).

To complicate the picture a number of empirical regularities have been detected. A mong them, B anz (1981) relates expected returns to ..rm size, Fama and French (1995) link expected returns to the ratio of book to market value. Some of these anomalies fade over time, other ones seem to be more persistent, rising the possibility that they are due to omitted systematic risk factors.

Pricing anomalies may be related to the possibility that useless factors appear to be priced. Of course it is also possible that pricing anomalies proxy for omitted factors. While statistical tests do not allow us to choose among these two possible explanations of pricing anomalies, K an and Zhang (1999a,b) suggest that perhaps large increase in $R^{2}$ and persistence of sign and size of coed cients over time are most likely to be associated with truly priced factors.

To investigate the exects of possible misspeci..cations of the return generating process on tests of asset pricing we study the portfolios used by J agannathan and Wang (1996). They are one hundred portfolios sorted by beta and size to maximize the spread of observed betas. To test the extent of misspeci..cation of the return generating process we nest the usual market model into the extended version used by Barone-A desi (1985).

The rest of the paper is organized as follows. Section 2 introduces the Quadratic Market Model (QM M). Section 3 brieły reviews estimation and inference methodologies traditionally used for asset pricing models of this kind. Section 4 reports empirical results, and section 5 concludes. 


\section{The Quadratic M arket M odel.}

The evolution of ..nancial asset returns shows signi..cant comovements. Factor models try to explain these comovements by a rather small number of underlying variables, called factors, which have a common exect on the returns dynamics.

The quadratic market model is an extension of the traditional market model, where market returns and the square of the market returns are the two factors.

The model speci..cation is:

$$
\begin{gathered}
R_{t}=a+B R_{M ; t}+i R_{M ; t}^{2}+{ }_{t} \\
E\left(" t j \underline{R_{M ; t}}\right)=0
\end{gathered}
$$

where $R_{t}$ is a $N \notin 1$ vector of asset returns in period $t, R_{M}$; $t$ is the return of the market in period $t$, $a$ is a $N £ 1$ vector of intercepts and $B$ and $i$ are $N £ 1$ vectors of sensitivities.

The motivation for including the square of the market returns is to account for risk originating from coskewness with the market portfolio. Speci..cally, the traditional market model postulates that asset returns move proportionally to the market. However, empirically we observe violations to this simple speci... cation. In fact, some classes of assets show a tendency, relatively to a linear dependence with a given market portfolio proxy, to have higher (lower) returns when the market experiences high absolute returns. Such an asset features positive (negative) coskewness and therefore diminishes (increases) the risk of the portfolio with respect to extreme events. See K raus and Litzenberger (1976), Barone-A desi (1985), Harvey and Siddique $(1999,2000)$.

In this sense, the quadratic market model captures possible non-linearities in the dependence between asset returns and market returns, as well as asymmetries in responses to upward and downward market movements.

As we will see more deeply in the empirical section, the misspeci..cation arising from neglecting these non-linearities and asymmetries can produce a signi..cant heterogeneity across portfolios. When the heterogeneity is correlated to variables representing portfolios characteristics such as size, these variables will appear to have explanatory power for the cross-section of expected returns. Taking into account the quadratic term, we are able to control for these heterogeneities.

This point addresses the problem linked to the fact that if there are similarities in the processes generating the data across and within the portfolio groups, combining the data improves the el ciency of the estimation of the parameters. There exists a huge body of literature in panel data econometrics pointing out the various consequences for inferences of neglecting heterogeneity (Robertson and Symons (1992), Pesaran and Smith (1995), H siao, Pesaran and Tahmiscioglu (1999), Haque, Pesaran and Sharma (1999), Pesaran, Shin and Smith (1999), Baltagi and Griф n (1997), Baltagi, Griф̧ n and Xiong (2000) amongst others). 
Usual arbitrage pricing considerations ${ }^{1}$, see Ross (1976), Chamberlain and Rothschild (1983), imply that expected returns of assets following the factor model (1) satisfy the restriction:

$$
E\left(R_{t}\right)=, 0+B, 1+i, 2
$$

where, 0 is the expected return on a portfolio for which the components ${ }^{-}$and ${ }^{\circ}$ of the vectors $\mathrm{B}$ and $\mathrm{i}$ are ${ }^{-}={ }^{\circ}=0$, while, 1 and , 2 are expected excess returns on portfolios perfectly correlated with $R_{M}$; and $R_{M ; t}^{2}$.

Since the risk-free asset and the ..rst factor, i.e. the market, satisfy (2), it must be that, $0=R_{F}$, the risk-free rate, and, $1=E\left(R_{M ; t}\right) ; R_{F}$. It is important to notice that a similar restriction doesn't hold for the second factor since it is not a traded asset, but it is possible to show (see Barone-A desi (1985)) that , $2<E\left(R_{M ; t}^{2}\right)$.

Equation (2) implies the restriction

$$
a=S_{N} R_{F} i B R_{F}+i\left(.2 i E\left(R_{M ; t}^{2}\right)\right)
$$

where $S_{N}$ is a $N \notin 1$ vector of ones, and imposing it on the factor model (1) we get

$$
R_{t} \text { i } S_{N} R_{F}=B\left(R_{M ; t} i R_{F}\right)+i^{i} R_{M ; t}^{2}+\#_{2}{ }^{\Phi}+{ }^{\prime} t
$$

where $\#_{2}=, 2$ i $E\left(R_{M ; t}^{2}\right)<0$.

Written for each portfolio separately we have:

$$
R_{\text {it i }} R_{F}={ }^{-}{ }_{i}\left(R_{M ; t} i R_{F}\right)+{ }^{0} R_{M ; t}^{2}+{ }_{i} \#_{2}+{ }^{\prime i t} \quad i=1 ;: ; ; N
$$

which is a nonlinear panel data model. It is worth noticing that the factors representing time varying regressors are common to all assets, whereas the equilibrium condition induce a restriction for the cross-section of expected returns through the term ${ }^{\circ}{ }_{i} \#_{2}$. This particularity explains why linear factor models of this kind are traditionally estimated by combinations of time series and crosssection regressions, as will be explained in the next section.

\section{Estimation and Inference}

In this section we present a general formulation of the QM M and then we discuss the estimation and inference procedures used for models of this kind .

The QMM presented in the section above is an example of a two-factor model, where one factor, denoted by $f_{1 ; t}$, is a return (the market), and the other, denoted by $f_{2 ; t}$, is not (it is the market squared return).

\footnotetext{
${ }^{1}$ Or an equilibrium model
} 
Thus, the unrestricted model (1) can be rewritten in more general form:

$$
\begin{aligned}
& R_{t}=a+B f_{1 ; t}+i f_{2 ; t}+{ }^{\prime} t
\end{aligned}
$$

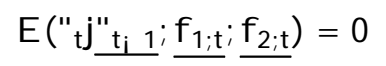

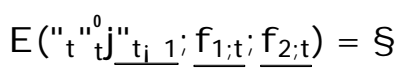

which corresponds to the null hypothesis in mixed form²:

$$
H_{0}^{(1)}: \quad 9 \#_{2}: a=\left(S_{N} i B\right) R_{F}+\#_{2 i}
$$

Additionally we may consider a more general restricted model

$$
R_{t}=S_{N} R_{F}+B\left(f_{1 ; t} i \quad R_{F}\right)+i f_{2 ; t}+i \#_{2}+t_{N}+{ }_{t}
$$

resulting from

$$
\mathrm{H}_{0}^{(2)}: \quad 9 \#_{2} ; \pm: \mathrm{a}=\left(\mathrm{S}_{\mathrm{N}} \mathrm{i} \quad \mathrm{B}\right) \mathrm{R}_{\mathrm{F}}+\#_{2 i}+ \pm \mathrm{S}_{\mathrm{N}}
$$

where \pm a scalar parameter.

The unrestricted factor model is represented by (3) and we refer to it as $H_{F}$. The pure equilibrium model is consistent with $\mathrm{H}_{0}^{(1)}$, whereas under $\mathrm{H}_{0}^{(2)}$ an additional constant is present in the cross-section of expected returns. We interpret the additional constant under $\mathrm{H}_{0}^{(2)}$ as representing omitted factors having an exect on expected returns which is homogeneous across portfolios.

Notice that $\mathrm{H}_{0}^{(1)} 1 / 2 \mathrm{H}_{0}^{(2)} 1 / 2 \mathrm{H}_{\mathrm{F}}$.

\subsection{Maximum Likelihood}

For the unrestricted model (3), it is well-known from the literature on seemingly unrelated regression (SUR) models that maximum likelihood (ML) estimation is equivalent to ordinary least squares (OLS) portfolio by portfolio (Zellner (1962)).

We now brieły present the $M L$ estimation of the restricted model $(5)^{3}$. We use the notation $F_{t}=\left(f_{1 ; t} ; f_{2 ; t}\right)^{0}$. Under the assumption of exogeneity

$$
\mathrm{F}_{\mathrm{t}} \mathrm{F}_{\mathrm{t}_{\mathrm{i}} 1} ; " \underline{\mathrm{t}_{\mathrm{i}} \mathrm{I}}{ } \gg\left({ }^{1} ; \S_{\mathrm{f}}\right)
$$

\footnotetext{
${ }^{2}$ To avoid unduly notational complexity, we indicate with 1 a column vector with unitary entries.

${ }^{3}$ T he estimation of (4) is derived from this by setting $\pm=0$.
} 
and joint normality of the " $t$, the restricted conditional loglikelihood function is:

$$
L=i \frac{N T}{2} \log 2 \frac{1}{4} / i \quad \frac{T}{2} \log \operatorname{det} \S i \frac{1}{2}_{t=1}{ }^{\top}{ }_{t}{ }_{t}(\mu)^{0} \S{ }^{i}{ }^{1 "}{ }_{t}(\mu)
$$

where

$$
{ }_{t}(\mu)=R_{t} \text { i } S_{N} R_{F} ; B\left(f_{1 ; t} \text { i } R_{F}\right) \text { i i } f_{2 ; t} \text { i } ; \#_{2} \text { i } \uplus_{N}
$$

and

$$
\mu=\left(\mathrm{B}^{0} ; \mathrm{i}^{0} ; \#_{2} ; \pm \operatorname{vec}(\S)^{0}\right)^{0}
$$

The maximum likelihood estimator is given by:

$$
\begin{aligned}
& \text { (由) } p={ }^{\tilde{A}} X\left(R_{t} ; R_{F} ; S_{N}\left(F_{t}+\frac{!}{0} \phi\right.\right. \\
& \tilde{A}^{t} X\left(F_{t}+F_{t}+m^{!^{i} 1}\right. \\
& \mathrm{t}
\end{aligned}
$$

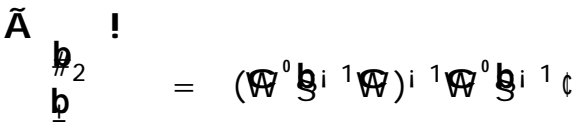

$$
\begin{aligned}
& : \frac{1}{T}^{\mu}\left(R_{t} i R_{F}\right) ; B^{\frac{1}{T}}{ }^{X}\left(f_{1 ; t} i R_{F}\right) ; p_{\frac{1}{T}}^{X} f_{2 ; t} \text { ๆ } \\
& \oint=\frac{1}{T}_{t}^{X} B_{t} B_{t}^{0}
\end{aligned}
$$

where

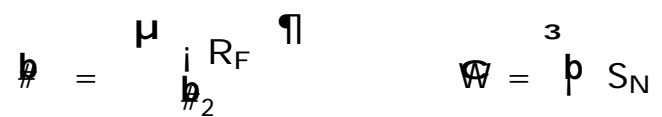

$$
\begin{aligned}
& b_{t}=R_{t} ; S_{N} R_{F} ; B\left(f_{1 ; t} ; R_{F}\right) ; p_{2 ; t} \quad p q_{2} ; S_{N}
\end{aligned}
$$

Therefore the sensitivities B and i are estimated by separate (asset-byasset) time-series regressions of excess returns on factors, whereas $\#_{2}$ and \pm are estimated by generalized least-squares (GLS) cross-section regressions of excess returns on $B, i$ and $S_{N}$.

These regressions are unfeasible since they would have to be run simultaneously. An iterative algorithm uses initial estimates for and to estimate B and $i$ from (6). The obtained values $B$ and $P$ are then used to update $\#_{2}$ and \pm with (7). 
The information matrix is block-diagonal in $\left(B ; i ; \#_{2} ; \sharp, \S\right.$. This implies that the ML estimators ( $B ; p ; p_{2} ;$, and $\$$ are asymptotically independent. We report here the asymptotic variances of $p,{ }_{2}$, and the will use later in the empirical part:

$$
\begin{aligned}
& \operatorname{AsVar}(P)=E^{3}\left(F_{t}+\#\left(F_{t}+\#\right)^{\prime} 22 \S+\right. \\
& +\S_{f}^{i 1}{ }_{2}{ }^{0} E^{3}\left(F_{t}+\#\left(F_{t}+\#\right)^{0} i 1^{9} W^{3} W^{0} \S^{i 1} W^{\prime}{ }^{i 1} W^{0}\right.
\end{aligned}
$$

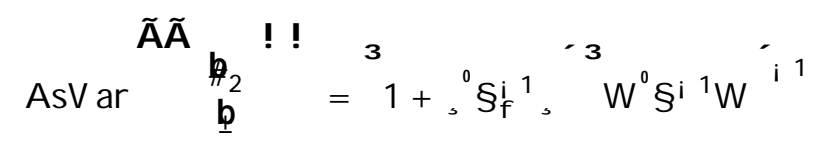

where $\mathrm{W}=\left(i \quad \mathrm{~S}_{\mathrm{N}}\right)$ and, $=(, 1 ;, 2)^{0}$.

We now consider the estimation of the risk premium, $2=\#_{2}+E\left(f_{2 ; t}\right)$ associated with the second factor. The ML estimator for, 2 is obtained by cross-sections GLS regression of excess returns on $B, i$ and $S_{N}$ :

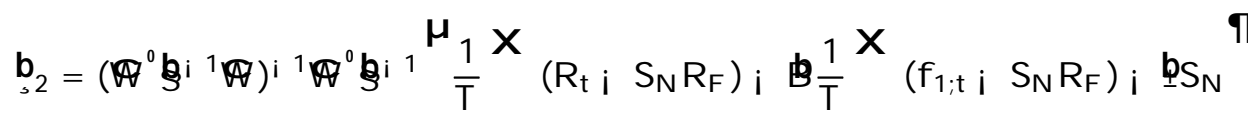

Its asymptotic variance is given by:

$$
\operatorname{AsVar}\left(b_{2}\right)={ }^{3} 1+,{ }^{0} \S_{f}^{i 1},{ }^{3}, W^{0} \S^{i 1} W^{\prime 11}+\S_{f ; 22}
$$

The hypothesis $\mathrm{H}_{0}^{(\mathrm{j})}, \mathrm{j}=1 ; 2$, can be tested against the general alternative $H_{F}$ by a likelihood ratio test. The test statistics is given by:

$$
\nu_{T}^{L R}=T\left(\log \left(\operatorname{det} \Phi_{0}\right) ; \log \left(\operatorname{det} \Phi^{\prime}\right)\right)
$$

where $\oiint_{0}$, resp. $\oint^{\circ}$, is estimated under $H_{0}^{(j)}$, resp. $H_{F}$. Under the null $H_{0}^{(j)}, \gg \iota^{R}$ is asymptotically $\hat{A}^{2}(q)$ where $q$ is the number of constraints imposed by $H_{0}^{(j)}$.

M oreover $\mathrm{H}_{0}^{(1)}$ can be tested against the alternative $\mathrm{H}_{0}^{(2)}$ by a simple asymptotic Wald-test based on \pm

\footnotetext{
${ }^{4}$ Upper indices refer to elements of the inverse matrix.
} 


\subsection{A symptotic Least Squares}

The hypotheses $\mathrm{H}_{0}^{(\mathrm{j})} \mathrm{j}=1 ; 2$ are of the mixed form and can be tested using asymptotic least squares (see Gourieroux, M onfort and Trognon (1985)), which provide an equivalent testing procedure to constrained $M L$.

Let $\mu=\left(a^{0} ; B^{0} ; i^{0} ; \operatorname{vec}(\S)^{0}\right)^{0}$ denote the parameters in the unconstrained model $\mathrm{H}_{\mathrm{F}}, \mathrm{b}=\left(\#_{2} ; \sharp^{0}\right.$ are the auxiliary parameters. The restriction $\mathrm{H}_{0}^{(2)}$ is $5^{5}$ :

$$
H_{0}^{(2)}: 9 \#_{2} ; \pm: g(\mu ; b)=0
$$

with $g(\mu ; b)=a_{i}\left(S_{N} ; B\right) R_{F} ; \quad \#_{2 i} i \quad S_{N}$.

A test statistic which is asymptotically equivalent to the likelihood ratio test (9) is:

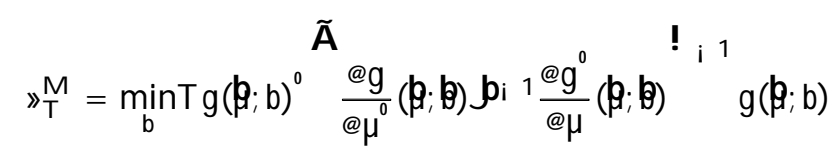

where $\mathrm{k}, \mathrm{q}$ and $\mathrm{J}$ are consistent estimators of the parameters $\mathrm{b}, \mu$ and of the information matrix. The solution to this problem, the asymptotic least square estimator, can be shown to be equivalent to the constrained MLE under the null hypothesis $\mathrm{H}_{0}^{(2)}$.

In this setting the problem reduces to:

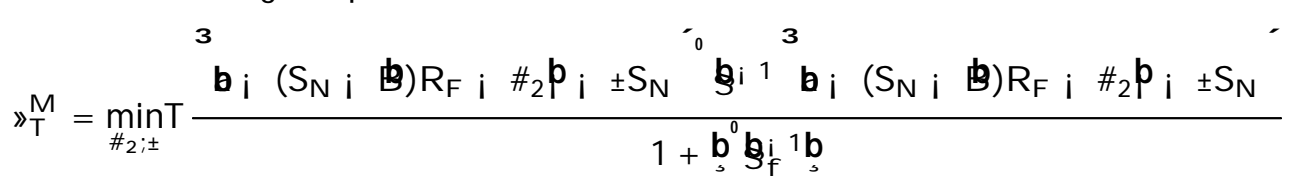

The solution ${ }_{2}$; to this minimization problem is found by using consistent estimates for $a, B, i, \S$ obtained by the estimation under $H_{F}$, whereas no estimate of , is needed, since it appears in a multiplicative factor which is irrelevant. For constructing the test statistic $\nu_{T}^{\mathrm{M}}$, we use $\#_{2}$ to estimate,

Therefore $\#_{2}$ apd \pm are estimated by $p^{a}$ GLS cross-section regression of $a_{i}$ $\left(S_{N} ;\right.$ B) $R_{F}=\frac{1}{T}\left(R_{t} ; S_{N} R_{F}\right) ;$ 皮 $\frac{1}{T}\left(f_{1 ; t} ; S_{N} R_{F}\right) ; p_{\frac{1}{T}} f_{2 ; t}$ on $P$ and $S_{N}$, which correspond to one step of the iterative $M L$ procedure.

The result and those derived for the $M L$ estimation justify the common practice in ..nance to use a two step procedure, where in the second step the factor risk premia are estimated by cross-section regressions of excess returns on estimated sensitivities obtained in the ..rst step. Shannken (1992) shows that the procedure of correcting the usual GLS variance $\mathrm{W}^{0} \S^{i}{ }^{1} \mathrm{~W}{ }^{11}$ with the factor $1+, \S_{f}^{i} 1$, - to take in account errors in the variable originating from

\footnotetext{
${ }^{5}$ We discuss here the case of $\mathrm{H}_{0}^{(2)}$. The case of $\mathrm{H}_{0}^{(1)}$ is similar.
} 
using estimated sensitivities - and of considering factor variability $\S_{f ; 22}$ leads to correct inference ${ }^{6}$.

\section{Empirical Results}

In this section we report the results from various empirical exercises, concerning the estimation of the QM M using the data set in J agannathan and Wang (1996), consisting of 330 monthly returns of 100 size- and beta-ranked portfolios for the period 1963-19907. We aggregate the 10 portfolios in each size decile in order to obtain 10 size-portfolios ${ }^{8}$, ranked in order of increasing size.

The following Tables report the main results. Table 1 contains the estimated ${ }_{i}$ from the unrestricted model $\mathrm{H}_{F}$ in (3) (t-statistic in parenthesis). In Table 2 we have estimates for the coet cients ${ }^{\circ}, \#_{2}, 2$ and \pm for the restricted model $\mathrm{H}_{0}^{(2)}$ in (5).

[Insert Table 1 about here]

[Insert Table 2 about here]

$>$ From the $B_{i}$ estimated in the unrestricted model, the quadratic market model itself seems to be a valuable extension of the basic market model, since the sensitivities to quadratic market returns appear signi..cantly negative for small ..rms and signi..cantly positive for large ..rms. We also notice that these estimates are similar to those obtained in the restricted model.

The ..ndings of a positive dependence of coskewness sensitivities to size in the period 1963-1990 are consistent with the results in Harvey and Siddique $(1999,2000)$.

The risk premium for coskewness, 2 is negative. In fact, portfolios which tend to have higher positive returns when the market has high absolute returns (positive coskewness) help the investor to reduce the risk for extreme events, and therefore command lower expected returns.

B eing the gammas of order $10^{i}{ }^{2} i 10^{i}{ }^{3}$, the part of expected excess returns due to coskewness is of order of a fraction of one percentage point.

\footnotetext{
${ }^{6} \mathrm{~T}$ hese correction factors hold under the assumption of errors uncorrelated through time.

${ }^{7}$ We use percentage monthly returns.

${ }^{8} \mathrm{~T}$ he basic motivation for doing this is to avoid estimation of variance-covariance matrices of large dimensions.
} 
$>$ From a statistical point of view, our estimates suggest that the pure equilibrium model based on a quadratic market model is rejected by data, being the constant term signi..cantly dixerent from zero. There is evidence that other components infuencing expected returns have been possibly omitted in the equilibrium model. The order of magnitude of the discrepancy we ..nd in monthly returns is approximately 40 basis points, on average across assets, which is relevant from an economic point of view.

These results are con..rmed by testing $\mathrm{H}_{0}^{(1)}$ against the general alternative $\mathrm{H}_{F}$. The test statistic is

$$
\nu_{T}^{M}=46: 73
$$

which is $\hat{A}^{2}(9)$ distributed and it is largely signi..cant at the 0:05 level.

In addition to assess the order of magnitude of the discrepancy that data show with the equilibrium model, it is interesting to investigate the degree of heterogeneity across assets of this discrepancy. At one extreme, the omitted components are the same for all portfolios, in terms of their contribution to expected returns. This situation is represented by $\mathrm{H}_{0}^{(2)}$, and we refer to it as the homogeneity hypothesis. At the opposite extreme, each portfolio shows a speci..c exect, and no restriction across portfolios' expected returns holds. This corresponds to $\mathrm{H}_{\mathrm{F}}$. In between, we can have situations where the omitted components are correlated to assets characteristics such as size, which cause these variables to appear signi..cative in the cross-section of expected returns.

Therefore the interest in the hypothesis of homogeneity is that it implies that additional ad hoc variables such as size have no further explanatory power once the term related to coskewness sensitivity is introduced.

A test of homogeneity can be conducted testing the null of $\mathrm{H}_{0}^{(2)}$ against the general alternative of $\mathrm{H}_{\mathrm{F}}$.

We can perform a likelihood ratio test:

$$
\nu_{T}^{\mathrm{R}}=\mathrm{T}\left(\log \left(\operatorname{det} \Phi_{0}\right) \mathrm{i} \log (\operatorname{det} \Phi)\right)
$$

where $\oint_{0}$ is estimated under $\mathrm{H}_{0}^{(2)}$ with $\mathrm{z}$ a constant, and $\oint$ from $\mathrm{H}_{\mathrm{F}}$. Under the Null, $\gg \uparrow^{R}$ is asymptotically $\hat{A}^{2}(8)$. The critical value at $\AA=0: 05$ is $\hat{A}_{0: 95}^{2}(8)=$ 15:51.

We obtain:

$$
\gg r^{\mathrm{L}}=12: 12
$$

The value computed above doesn't reject the hypothesis of homogeneity.

Similar results are obtained using asymptotic least-squares, with a test statistic for $\mathrm{H}_{0}^{(1)}$ against the general alternative $\mathrm{H}_{\mathrm{F}}$ equal to

$$
\nu_{T}^{M}=11: 49
$$

which is not signi..cant at the 0:05 level. 
Therefore, there is evidence for homogeneity in the quadratic market model. We are interested to assess the importance of the quadratic term for controlling heterogeneity in the intercepts of the portfolios.

To this aim, we investigate the improvement over the traditional market model obtained using the extended quadratic version. The traditional market model is characterized by the factor model (3) with $i=0$. The CAPM implies the restriction $a=(1 ; B) R_{F}$.

We compute the asymptotic least-squares statistic $\nu_{T}^{M}$ for testing

$$
\mathscr{\Phi}_{0}^{(2)}: 9 \pm: a_{i}(1 ; \quad B) R_{F}= \pm S_{N}
$$

The result is:

$$
\nu_{T}^{M}=23: 14
$$

which is larger than the critical value at 0:05, $\hat{A}_{0: 95}^{2}(9)=16: 90$.

Neglecting the quadratic term, there is signi...cative evidence for heterogeneity across portfolios. At an explorative level, it is instructive to analyze the

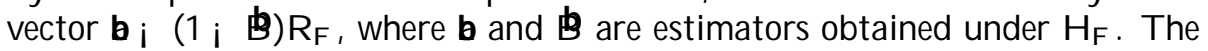
components corresponding to small size portfolios are higher than those of larger portfolios by approximately half of a percentage point. The heterogeneity appears therefore to be negatively correlated to size. The introduction of the quadratic term helps considerably in controlling the heterogeneity in the intercepts, since the term $\#_{2 i}$ too is negatively correlated to size.

\section{Conclusions}

In this paper we consider a two factor model to study the presence of significant comovements in the dynamics of asset returns. In addition to the traditional market returns term the model includes the square of the market returns to account for risk originating from coskewness with the market portfolio as recent literature (see for instance Harvey and Siddique (2000)) also stresses. In addition, we showed that the quadratic term is able to account for heterogeneities across portfolios otherwise improperly controlled via a simple constant. We show that the extra term is signi..cant and that the homogeneity hypothesis is accepted only in the presence of this term.

Although there is evidence that QMM is not a complete description of asset pricing, acceptance of homogeneity is supportive of the signi..cance of its contribution in explaining security returns. 


\section{A ppendix}

TABLE 1

\begin{tabular}{|c|c|c|c|c|}
\hline $\begin{array}{r}B_{1}=\underset{i}{i} 0: 014 \\
(i, 1: 99)\end{array}$ & 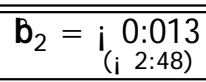 & $\begin{array}{r}B_{3}=\underset{i}{i} 0: 010 \\
(i, 2: 09)\end{array}$ & $\begin{array}{r}\mathrm{B}_{4}=\underset{i}{\mathrm{i}} 0: 010 \\
(\mathrm{i} 2: 28)\end{array}$ & 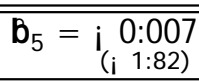 \\
\hline 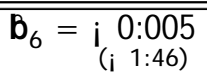 & 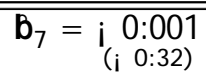 & 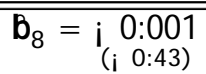 & 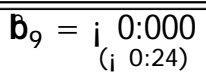 & $\begin{array}{c}B_{10}=0: 003 \\
(2: 09)\end{array}$ \\
\hline
\end{tabular}

TABLE 2

\begin{tabular}{|c|c|c|c|c|}
\hline$B_{1}=\underset{i}{i} 0: 015$ & $B_{2}=\underset{i}{i} 0: 012$ & $B_{3}=\underset{i}{i} 0: 0099$ & $B_{4}=\underset{i}{i} 0: 0100$ & $B_{5}=\underset{\substack{i \\
(i}}{0: 0063)}$ \\
\hline 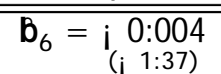 & $\mathrm{B}_{7}=\underset{i \underset{i}{0} 0: 000}{0: 17)}$ & $\overline{B_{8}=\underset{(i}{i} 0: 0001}$ & $\begin{array}{c}B_{9}=0: 001 \\
(0: 46)\end{array}$ & $\begin{array}{c}0: 004 \\
\left.B_{10}=0: 12\right) \\
(3: 12)\end{array}$ \\
\hline 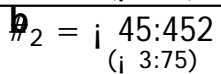 & $\mathrm{b}_{2}=\mathrm{i}_{(\mathrm{i}} 24: 416$ & $\underline{\underline{q}}=0: 416$ & & \\
\hline
\end{tabular}




\section{R eferences}

[1] Baltagi,B.H. and J.M.Griф n (1997), "Pooled Estimators versus their Hetergenous Counterparts in the Context of Dynamic Demand for Gasoline", J ournal of Econometrics, 77, 303-27.

[2] Baltagi,B.H., J.M.Griф n, and W.Xiong (2000), "To Pool or not to Pool: Homogenous versus Heterogenous Estimators Applied to Cigarette Demand", R eview of E conomics and Statistics, 82, 117-26.

[3] Banz, R. W . (1981), "The relationship between Return and Market Value of Common Stocks", J ournal of Financial Economics, 9, 3-18.

[4] Barone-A desi, G. (1985), "A rbitrage Equilibrium with Skewed Asset Returns", J ournal of F inancial and Quantitative Analysis, 20, 299-313.

[5] Black, F. (1972), "Capital Market Equilibrium with Restricted Borrowing", J ournal of Business, 45, 444-55.

[6] Breeden, D. T. (1979), "An Intertemporal Asset Pricing Model with Stochastic Consumption and Investment Opportunities", J ournal of Financial E conomics, 7, 265-96.

[7] Campbell, J . Y . (2000), "A sset Pricing at the Millenium", forthcoming in J ournal of Finance.

[8] Cochrane, J. H. (1996), "A Cross-sectional Test of an Investment-based A sset Pricing M odel", J ournal of Political Economy, 104, 572-621.

[9] Fama, E. and K. R. French (1995), "Size and B ook-to-Market Factors in E arnings and R eturns", J ournal of F inance, 50, 131-55.

[10] Gourieroux, C., A. Monfort, and A. Trognon (1985), "Moindres Carrés A symptotiques", A nnales de l'INSE E, 58, 91-122.

[11] Haque,N.U., M.H. Pesaran and S.Shama (1999), "Neglected Heterogeneity and Dynamics in Cross-Country Saving Regressions", forthcoming in J.Krisnakumar and E.Ronchetti (eds) Panel Data Econometrics: Future Directions: Papers in Honour of Prof. Balestra, Elsevier Science.

[12] Harvey,C.R. and A. Siddique (1999), "A utoregressive Conditional Skewness", J ournal of F inancial and Quantitative A nalysis, 34, 465-487.

[13] Harvey,C.R. and A. Siddique (2000), "Conditional Skewness in A sset Pricing Tests", J ournal of Finance, 55,1263-1295.

[14] Hsiao,C., M.H. Pesaran and A.K. Tahmiscioglu (1999), "Bayes Estimation of Short-Run Coec cients in Dynamic Panel Data Models", in Analysis of Panels and Limited Dependent Variable Models: A Volume in Honour of G.S.Maddala edited by C.Hsiao,K.Lahiri,L-F Lee and M.H. Pesaran, Cambridge: Cambridge University Press. 
[15] J agannathan, R. and Z. Wang (1996), "The Conditional CAPM and the Cross-Section of Expected Returns" J ournal of Finance, 51, 3-53.

[16] K an, R. and C. Zhang (1999a), "GM M Test of Stochastic Discount Factor M odels with Useless Factors", J ournal of F inancial Economics, 54, 103-27.

[17] Kan, R. and C. Zhang (1999b), "T wo-Pass Tests of A sset Pricing M odels with U seless Factors", J ournal of F inance, 54, 203-35.

[18] K raus, A . and R. Litzenberger (1976), "Skewness Preferences and the Valuation of Risk A ssets", J ournal of Finance, 31, 1085-1100

[19] Lintner, J . (1965), "The Valuation of Risk Assets and the Selection of Risky Investments in Stock Portfolios and Capital Budgets", Review of E conomics and Statistics, 47, 13-37.

[20] Merton, R. C. (1973), "An Intertemporal Capital Asset Pricing Model", E conometrica, 41, 867-87.

[21] Pesaran,M.H., Y.Shin, and R.P. Smith (1999), "Bounds Testing Approaches to the A nalysis of Long-R un Relationships", University of Cambridge, DAE Working Paper 9907.

[22] Pesaran,M.H. and R.P. Smith (1995), "Estimating Long-R un R elationships from Dynamic Heterogenous Panels", J ournal of Econometrics, 68, 79-113

[23] Robertson,D. and J.Symons (1992), "Some Strange Properties of Panel Data Estimators", j ournal of A pplied E conometrics, 7, 175-89.

[24] Ross, S. A. (1976), "A rbitrage Theory of Capital A sset Pricing", J ournal of E conomic Theory, 13, 341-60.

[25] Shanken, J . (1992), "On the Estimation of B eta Pricing Models", Review of Financial Studies, 5, 1-33.

[26] Sharpe, W . F . (1964), "Capital A sset Prices: A Theory of Market Equilibrium Under Conditions of Risk " , J ournal of F inance, 40, 1189-96.

[27] Zellner, A. (1962), "A n E \$ cient M ethod of Estimating Seemingly Unrelated Regressions and Tests for Aggregation Bias", J ournal of A merican Statistical Association, 57, 348-68. 